\title{
Actual Metal Coverage at the Neck Is Critical for Flow- Diverting Stents in Treating Intracranial Aneurysms
}

W

e read the interesting article by Schneiders et al, ${ }^{1}$ entitled "A Flow-Diverting Stent Is Not a Pressure-Diverting Stent." The authors measured intra-aneurysmal pressure before, during, and after placement of a flow-diverting (FD) stent and found that the pressure inside the aneurysm momentarily decreased during placement but was restored to baseline values within minutes. They agreed with the argument that the use of an FD stent for treatment of intracranial aneurysms should be combined with insertion of coils in the aneurysmal sac. After carefully observing the conventional angiogram in their article, we found that the included angle of the radiopaque longitudinal markers of the stent at the neck was approximately $60^{\circ}$. Therefore, the stent was partly elongated at the neck of the aneurysm.

The metal coverage rate (MCR) of an FD stent can change as a result of either compression or stretching. The MCR $(\zeta)$ and pore density of the stent can be calculated according to the following formula:

$$
\begin{gathered}
\zeta=\left[1-\left(\frac{N \cdot d}{B \cdot \sin \alpha}\right)^{2}\right] \times 100 \% \\
\text { Pore Density }=\frac{N^{2}}{B^{2} \cdot \sin \alpha},
\end{gathered}
$$

where $N$ represents the wire number, $d$ stands for the wire diameter of the stent, $B$ represents the length of the wire, which spirals a complete turn, and $\alpha$ represents the included angle between wires along the long axis of the stent. All measured units are in millimeters. The tendency of the MCR corresponding to the included angle of the stent wires is shown in Fig 1 when an FD stent is compressed or stretched.

An FD stent with an MCR of 30\% significantly affects the hemodynamics in the aneurysm. An FD stent with a 35\% actual MCR at the neck can predict $>95 \%$ of angiographic aneurysm occlusions in rabbits. ${ }^{2}$ The Pipeline Embolization Device (PED; Chestnut Medical Technologies, Menlo Park, California) provides a $30 \%-35 \% \mathrm{MCR}$ with approximately a $142^{\circ}-150^{\circ}$ nominal

http://dx.doi.org/10.3174/ajnr.A3451 included angle between wires when it is fully expanded. ${ }^{3}$ The Silk flow diverter (SFD; Balt Extrusion, Montmorency, France) provides 35\%-55\% MCR of the internal diameter of the target vessel at a nominal diameter, and the included angle between wires is $>120^{\circ}$, as shown by a previous study. ${ }^{4}$ In an in vitro study of SFD morphology, the local actual MCR of the FD stent at different curvatures in different vessel models can change from 19\% to $63 \% .^{5}$ The MCR decreases to a minimum, which is only approximately $20 \%$, when the included angle decreases to $90^{\circ}$ in an FD stent as shown in Fig 1. This actual MCR at the neck may not significantly change hemodynamics. Even though the included angle between wires can enhance the MCR when decreased to $<90^{\circ}$, the stent is severely stretched; this change may cause the stent not to open.

The proper morphology of an FD stent at the neck is when the included angle between wires is equal to or higher than its nominal angle, where the local actual MCR can achieve or exceed 35\%. In vivo and in vitro studies have also indicated that properly pushing the microguidewire or microcatheter can gain more MCR at the neck for stasis and thrombosis of aneurysms. ${ }^{2,3}$ The included

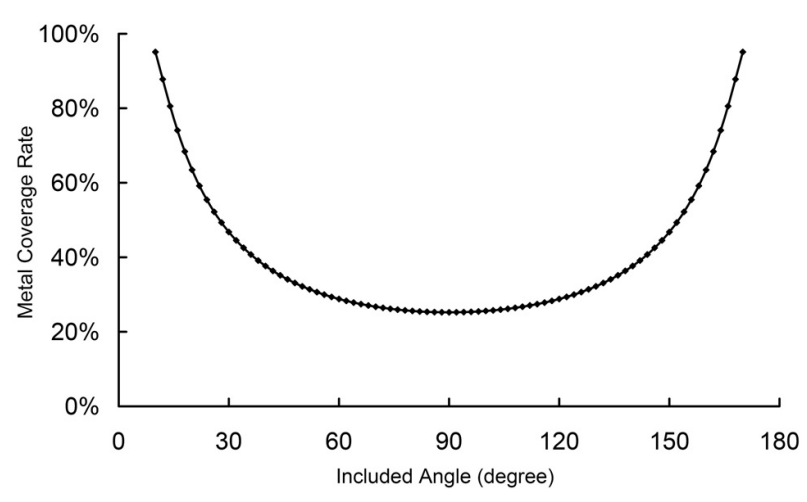

FIG 1. Correlation of the MCR with the included angle between wires along the long axis of an FD stent. This stent was made of 48 wires $(N)$ with a wire diameter of $0.04 \mathrm{~mm}(d)$ and a wire spiraling a complete turn length of $14.2 \mathrm{~mm}(B)$. When the included angle $(\alpha)$ is $90^{\circ}$, the MCR decreases to a minimum of approximately $20 \%$. When the included angle is $<30^{\circ}$ (eg, the stent is stretched in the microcatheter) or $>150^{\circ}$ (eg, the stent is compressed at the neck), the MCR sharply increases. 
angle between wires of an FD stent at the aneurysm neck can be easily displayed by angiographic CT.

\section{REFERENCES}

1. Schneiders JJ, Vanbavel E, Majoie CB, et al. A flow-diverting stent is not a pressure-diverting stent. AJNR Am JNeuroradiol 2013;34:E1-4

2. Wang K, Huang Q, Hong B, et al. Correlation of aneurysm occlusion with actual metal coverage at neck after flow-diverting stent implanted in rabbit models. Neuroradiology 2012;54:607-13

3. Ma D, Dargush GF, Natarajan SK, et al. Computer modeling of deployment and mechanical expansion of neurovascular flow di- verter in patient-specific intracranial aneurysms. $J$ Biomech 2012;45:2256-63

4. Kulcsár Z, Ernemann U, Wetzel SG, et al. High-profile flow diverter (Silk) implantation in the basilar artery: efficacy in the treatment of aneurysms and the role of the perforators. Stroke 2010;41:1690-96

5. Aurboonyawat T, Blanc R, Schmidt P, et al. An in vitro study of Silk stent morphology. Neuroradiology 2011;53:659-67

Department of Neurosurgery General Hospital of Jinan Military Command Jinan, China 